\title{
Squashed States of Light: Theory and Applications to Quantum Spectroscopy
}

\author{
Running Title: Quantum Spectroscopy with Squashed Light
}

\author{
H. M. Wiseman \\ Centre for Laser Science, The Department of Physics, \\ The University of Queensland, Queensland 4072 Australia. *
}

\begin{abstract}
Using a feedback loop it is possible to reduce the fluctuations in one quadrature of an in-loop field without increasing the fluctuations in the other. This effect has been known for a long time, and has recently been called "squashing" [B.C. Buchler et al., Optics Letters 24, 259 (1999)], as opposed to the "squeezing" of a free field in which the conjugate fluctuations are increased. In this paper I present a general theory of squashing, including simultaneous squashing of both quadratures and simultaneous squeezing and squashing. I show that a twolevel atom coupled to the in-loop light feels the effect of the fluctuations as calculated by the theory. In the ideal limit of light squeezed in one quadrature and squashed in the other, the atomic decay can be completely suppressed.
\end{abstract}

\section{Introduction}

Squeezed states of light are nonclassical [1]. The foremost consequence of this is that they can produce a homodyne photocurrent having a noise level below the shot-noise limit. The shot-noise limit is what is predicted by a theory in which the light is classical, with no noise, but the process of photo-electron emission is treated quantum-mechanically.

There is, however, a simple way to produce a sub-shot-noise photocurrent without squeezed light: modulating the light incident on the photodetector by a current originating from that very detector. This was first observed [2, 3] around the same time as the first incontestable observation of squeezing [4].

The sub-shot noise spectrum of an in-loop photocurrent is not regarded as evidence for squeezing for a number of reasons. First, the two-time commutation relations for an in-loop field are not those of a free field [5]. This means that it is possible to reduce the fluctuations in the measured (amplitude) quadrature without increasing those in the other (phase) quadrature. Second, attempts to remove some of the supposedly low-noise light by a beam splitter yields only above shot-noise light, as verified experimentally [2, 6].

Because of these differences, the presence of a sub-shot noise photocurrent spectrum for inloop light has by and large been omitted from discussions of squeezing [7, 8, 97. Nevertheless, it has been argued [10] that the in-loop field can justifiably be called "sub-Poissonian" (even if not squeezed), because a perfect quantum-non-demolition (QND) intensity meter for the in-loop light would register the same sub-shot-noise statistics as the (perfect) in-loop detector. Furthermore, it was proposed in Ref. [10] that the apparent in-loop noise reduction could be used to improve the signal to noise ratio for a measurement of a modulation in the coupling coefficient of such a QND intensity meter.

Following on from Ref. [10], it has been shown that in-loop optical noise suppression may have other, more practical, applications. Buchler et al. showed [11 that such in-loop light can suppress radiation pressure noise in a gravitational wave detector by a factor of two. Even more strikingly,

*E-mail: wiseman@physics.uq.edu.au. 
I recently showed [12] that a two-level atom coupled to the in-loop field can exhibit linewidth narrowing exactly analogous to that produced by squeezed light [13].

In this paper I will discuss the properties of "squashed" states of light, as the in-loop analogues of squeezed states of light are called in Ref. [11]. Section 3 covers the general theory of squashed states of light, including states which are squashed in both quadratures and states which are simultaneously squashed and squeezed. In Section 4, I generalize the analysis of Ref. [12] by considering the effects of these more general state of light on a two-level atom. But to begin, I review the properties of squeezed states of light in the following section.

\section{Squeezed States}

\section{$2.1 \quad$ Single-mode squeezing}

The noise in the quadratures of a single-mode light field of annihilation operator $a$,

$$
x=a+a^{\dagger}, \quad y=-i a+i a^{\dagger},
$$

is limited by the Heisenberg uncertainty relation

$$
V_{x} V_{y} \geq\left|\frac{1}{2}[x, y]\right|^{2}=1,
$$

where $V$ denotes variance. Squeezed states of light are states such that one of the quadrature variances, say $V_{x}$, is less than one 11, 14, 15. Clearly the other quadrature variance, say $V_{y}$, must be greater than one. If the equality is attained in the uncertainty relation then these are called minimum uncertainty squeezed states. The only other sort of minimum uncertainty state is the coherent state with $V_{x}=V_{y}=1$.

\subsection{Continuum Squeezing}

Single-mode squeezing was generalized early to multimode squeezing [16]. In this work I wish to consider the limit of an infinite number of modes: the electromagnetic continuum. Considering polarized light propagating in one direction, only a single real-valued index is needed for the modes, and we take that to be the mode frequency $\omega=k$ (using units such that the speed of light is one). Then the continuum field operators $b(k)$ obey

$$
\left[b(k), b^{\dagger}\left(k^{\prime}\right)\right]=\delta\left(k-k^{\prime}\right) .
$$

For light restricted in frequency to a relatively narrow bandwidth $B$ around a carrier frequency $\Omega$ it is possible to convert from the frequency domain to the time or distance domain by defining

$$
b(t)=\int_{\Omega-B}^{\Omega+B} d k b(k) e^{-i(k-\Omega) t} .
$$

These obey

$$
\left[b(t), b^{\dagger}\left(t^{\prime}\right)\right]=\delta\left(t-t^{\prime}\right),
$$

where $b^{\dagger}(t) \equiv[b(t)]^{\dagger}$, and the $\delta$ function is actually a narrow function with width of order $B^{-1}$. The operator $b^{\dagger}(t) b(t)$ can be interpreted as the photon flux operator.

Defining continuum quadrature operators

$$
X(t)=b(t)+b^{\dagger}(t), \quad Y(t)=-i b(t)+i b^{\dagger}(t),
$$

one obtains

$$
\left[X(t), Y\left(t^{\prime}\right)\right]=2 i \delta\left(t-t^{\prime}\right) .
$$

The singularity in the associated uncertainty relations can be avoided by quantifying the uncertainty by the spectrum 8

$$
S^{X}(\omega)=\langle\tilde{X}(\omega) X(0)\rangle_{\mathrm{ss}}-\langle\tilde{X}(\omega)\rangle_{\mathrm{ss}}\langle X(0)\rangle_{\mathrm{ss}} .
$$


Then one can derive the finite uncertainty relations [5]

$$
S^{X}(\omega) S^{Y}(\omega) \geq 1
$$

For very high frequencies the spectra always go to unity. This represents the shot-noise or vacuum noise level. However for finite frequencies it is possible to have for example $S_{X}(\omega)<1$. This indicates squeezing of the $X$ quadrature. The uncertainty in the conjugate quadrature would of course be increased.

The quadrature operators $X(t), Y(t)$ can be directly measured (one at a time) using homodyne detection [17]. For detection of efficiency $\epsilon \leq 1$ the photocurrent $I_{\text {hom }}^{X}$ has the same statistics as (and therefore can be represented by) the operator

$$
I_{\text {hom }}^{X}(t)=\sqrt{\epsilon} X(t)+\sqrt{1-\epsilon} \xi_{X}(t),
$$

where $\xi_{X}(t)$ is a Gaussian white noise term. Here the normalization has been chosen so that the photocurrent spectrum

$$
S_{\mathrm{hom}}^{X}(\omega)=\epsilon S^{X}(\omega)+(1-\epsilon),
$$

remains equal to unity (the shot noise limit) at high frequencies, where $S^{X}(\omega)=1$.

\subsection{An Atom in a Squeezed Bath}

Now consider the situation where a two-level atom is immersed in a beam of squeezed light with annihilation operator $b_{0}(t)$. If the degree of mode matching of the squeezed light to the atom's dipole radiation mode is $\eta$, then the dipole coupling Hamiltonian in the rotating-wave approximation is

$$
H_{0}(t)=-i\left[\sqrt{\eta} b_{0}(t)+\sqrt{1-\eta} \nu(t)\right] \sigma^{\dagger}(t)+\text { H.c. }
$$

Here $\sigma$ is the atomic lowering operator and the atomic linewidth has been set to unity. The operator $b_{0}(t)$ represents the squeezed field with spectra $S_{0}^{X}(\omega)$ and $S_{0}^{Y}(\omega)$, and $\nu(t)$ represents the vacuum field interacting with the atom, satisfying $\left\langle\nu(t) \nu^{\dagger}\left(t^{\prime}\right)\right\rangle=\delta\left(t-t^{\prime}\right)$.

Now if the quadrature spectra of the squeezed light are much broader than the atomic linewidth then one can make the white noise approximation that they are constant. For minimum-uncertainty squeezing, one has

$$
S_{0}^{X}(\omega)=L=1 / S_{0}^{Y}(\omega),
$$

and the atom will obey the master equation 13

$$
\dot{\rho}=(1-\eta) \mathcal{D}[\sigma] \rho+\frac{\eta}{4 L} \mathcal{D}\left[(L+1) \sigma-(L-1) \sigma^{\dagger}\right] \rho,
$$

where $\mathcal{D}[A] B \equiv A^{\dagger} B A-\frac{1}{2}\left\{A^{\dagger} A, B\right\}$ as usual. This leads to the following atomic dynamics:

$$
\begin{aligned}
\operatorname{Tr}\left[\dot{\rho} \sigma_{x}\right] & =-\gamma_{x} \operatorname{Tr}\left[\rho \sigma_{x}\right], \\
\operatorname{Tr}\left[\dot{\rho} \sigma_{y}\right] & =-\gamma_{y} \operatorname{Tr}\left[\rho \sigma_{y}\right], \\
\operatorname{Tr}\left[\dot{\rho} \sigma_{z}\right] & =-\gamma_{z} \operatorname{Tr}\left[\rho \sigma_{z}\right]-C,
\end{aligned}
$$

where

$$
\begin{aligned}
& \gamma_{x}=\frac{1}{2}[(1-\eta)+\eta L], \\
& \gamma_{y}=\frac{1}{2}\left[(1-\eta)+\eta L^{-1}\right], \\
& \gamma_{z}=\gamma_{x}+\gamma_{y}, \quad C=1 .
\end{aligned}
$$

Note that for $L<1$ the decay rate of the $x$ component of the atomic dipole is reduced below the vacuum level of $\frac{1}{2}$, while the decay rate of the other component is increased. The reduction or increase in the decay rates are directly attributable to the reduction or increase in the fluctuations of the respective quadrature of the input continuum field. The prediction of this effect by Gardiner [13] began the study of quantum spectroscopy (that is, the interaction of nonclassical light with matter) 18. 
For sufficiently large $\eta$ this effect would be easily detectable experimentally in the fluorescence power spectrum of the atom (into the vacuum modes):

$$
\begin{aligned}
P(\omega) & =\frac{1-\eta}{2 \pi}\left\langle\tilde{\sigma}^{\dagger}(-\omega) \sigma(0)\right\rangle_{\mathrm{ss}} \\
& =\frac{(1-\eta)\left(\gamma_{z}-C\right)}{8 \pi \gamma_{z}}\left[\frac{\gamma_{x}}{\gamma_{x}^{2}+\omega^{2}}+\frac{\gamma_{y}}{\gamma_{y}^{2}+\omega^{2}}\right] .
\end{aligned}
$$

For $L<1$ the spectrum consists of two Lorentzians, one with a sub-natural linewidth and one with a super-natural linewidth. The overall linewidth (defined as the full-width at half-maximum height) is reduced.

This line-narrowing is only noticeable if the degree of mode matching $\eta$ of the squeezed light to the atom is significant, which is hard to do with a squeezed beam. One way around this is to make the atom strongly coupled to a microcavity, which can be driven by a squeezed beam. The microcavity enhances the atomic decay rate, but a squeezed input should suppress this enhancement in one quadrature. Unfortunately, experiments to date have failed to see this suppression, due to imperfections of various kinds [19].

\section{Theory of Squashed States}

\subsection{Generation of Squashed States}

Consider the feedback loop shown in Fig. 1. The field entering the modulator is $b_{0}(t)=\frac{1}{2}\left[X_{0}(t)+\right.$ $i Y_{0}(t)$. The quadrature operators are assumed to have independent statistics defined by the spectra $S_{0}^{X}(\omega), S_{0}^{Y}(\omega)$. The modulator simply adds a coherent amplitude to this field. There are various ways of achieving this, one of which is discussed in Ref. 12. The field exiting is in any case given by

$$
b_{1}(t)=\frac{1}{2}\left[X_{0}(t)+i Y_{0}(t)+\chi(t)+i v(t)\right],
$$

where $\chi(t), v(t)$ are real functions of time. This field now enters a homodyne detection device, set up so as to measure the $X$ quadrature. If the efficiency of the measurement is $\epsilon_{X}$ then the photocurrent is given by

$$
I_{\text {hom }}^{X}(t)=\sqrt{\epsilon_{X}}\left[X_{0}(t)+\chi(t)\right]+\sqrt{1-\epsilon_{X}} \xi_{X}(t) .
$$

Now, through the feedback loop, this current may determine the classical field amplitudes $\chi, v$. Obviously in the case of measuring the $X$ quadrature, the only interesting results will come from controlling $\chi(t)$, and we set

$$
\chi(t)=\int_{0}^{\infty} g_{X} h(s) I_{\mathrm{hom}}^{X}(t-\tau-s) / \sqrt{\epsilon_{X}} d s .
$$

Here $\tau$ is the minimum delay in the feedback loop, $h(s)$ is the feedback loop response function normalized to $\int_{0}^{\infty} h(s) d s=1$ and $g_{X}$ is the round-loop gain [20]. Taking the fourier transform of this expression and substituting into Eqs. (23) and (24) yields

$$
\tilde{b}_{1}(\omega)=\frac{1}{2}\left[\frac{\tilde{X}_{0}(\omega)+\sqrt{\theta_{X}} g_{X} e^{i \omega \tau} \tilde{h}(\omega) \tilde{\xi}_{X}(\omega)}{1-g_{X} e^{i \omega \tau} \tilde{h}(\omega)}+i \tilde{Y}_{0}(\omega)+i v(t)\right] .
$$

where

$$
\theta_{X} \equiv \epsilon_{X}^{-1}-1
$$

The $X$ quadrature spectrum of this light is

$$
S_{1}^{X}(\omega)=\frac{S_{0}^{X}(\omega)+\theta_{X} g_{X}^{2}|\tilde{h}(\omega)|^{2}}{\left|1-g_{X} e^{i \omega \tau} \tilde{h}(\omega)\right|^{2}} .
$$

Say we are only interested in frequencies well inside the bandwidth of $\tilde{h}(\omega)$, much less than $\tau^{-1}$, and much less than the bandwidth of $S_{0}^{X}(\omega)$. Then we can replace $e^{i \omega \tau} \tilde{h}(\omega)$ by unity and $S_{0}^{X}(\omega)$ by a constant $L$. This gives

$$
S_{1}^{X}=\frac{L+g_{X}^{2} \theta_{X}}{\left(1-g_{X}\right)^{2}} \geq \frac{L}{1+L / \theta_{X}}
$$


where the minimum is achieved for negative feedback $g_{X}=-L / \theta_{X}$.

Evidently this minimum is less than $L$. This means that even starting with shot-noise limited light $(L=1)$ it is possible to produce sub-shot-noise light. However, it is important to note that this is not squeezed light in the ordinary sense. For example, it is impossible to remove any of the squeezed light by putting a beam splitter in the path of the in-loop beam. Under the above conditions the resulting out-of-loop beam actually has a noise level above the shot noise [2, 6, 5, 10]. Nevertheless, the fluctuations of the in-loop light do produce genuine physical effects in other circumstances, as investigated in Sec. 4.

\subsection{Violation of the Uncertainty Relations}

A curious point in the apparent squeezing of the $X$ quadrature is that the feedback has no effect on the $Y$ quadrature of $b_{1}$. From Eq. (26), $S_{1}^{Y}=L^{-1}$, assuming a minimum uncertainty input $b_{0}$ and $v=0$. Thus the uncertainty relation (9) is violated for this in-loop light. For this reason, the in-loop light exhibiting sub-shot-noise fluctuations has been called "squashed light" [11. By feedback, the noise in one quadrature can be squashed (reduced), but there is no "squeezing" of phase-space area into an increased noise in the other quadrature.

The reason that the uncertainty relation (9) is violated is that the commutation relations (5) are no longer valid for time differences $\left|t-t^{\prime}\right|>\tau$, the minimum feedback loop delay. This is a direct consequence of the feedback loop. It is important to realize that the parts of the inloop field separated in time by greater than $\tau$ never actually exist together. That is because the propagation time from the modulator to the detector is necessarily less than $\tau$. Thus the fundamental commutation relations [7] between parts of the field at different points in space at the same time are never violated.

For freely propagating fields there is no real distinction between space and time separations, but for an in-loop field it is a crucial distinction. The temporal anticorrelations in the in-loop squeezed light only exist for time separations greater than $\tau$, and hence greater than the time for which any part of the in-loop light exists. There is never any anticorrelation between parts of the in-loop field in existence at any given time. By contrast, conventional squeezed light can propagate for an arbitrarily long time before detection, so the anticorrelations are between parts of the field which can exist simultaneously (even if they may not actually do so in a given experiment).

\subsection{Simultaneous Squashing in Both Quadratures}

It is interesting now to consider feedback in both the $X$ and $Y$ quadratures. Obviously one cannot simultaneously measure both of these quadratures with unit efficiency, but one can measure $Y$ with efficiency $\epsilon_{Y} \leq 1-\epsilon_{X}$. Carrying through the same sort of analysis as above shows that the $Y$ quadrature spectrum can be simultaneously reduced to

$$
S_{1}^{Y}=\frac{L^{-1}}{1+L^{-1} / \theta_{Y}} .
$$

For the special case of $L=1$ (a vacuum input), one finds

$$
S_{1}^{Y}+S_{1}^{X}=2-\epsilon_{X}-\epsilon_{Y} \geq 1 .
$$

For feedback based on heterodyne detection (which is equivalent to homodyne detection on both

quadratures with equal efficiency), one can have $S_{1}^{Y}=S_{1}^{X}=1-\frac{1}{2} \epsilon$, which goes to one half in the limit of perfect detectors.

\subsection{Simultaneous Squeezing and Squashing}

In the general case of $L \neq 1$, the sum of the quadrature spectra need not even be greater than one. Rather, for fixed $L<1$ and fixed $\epsilon=\epsilon_{X}+\epsilon_{Y}$ one finds, for $\epsilon_{X}=0$ and $\epsilon_{Y}=\epsilon$

$$
S_{1}^{Y}+S_{1}^{X}=L+\frac{\epsilon^{-1}-1}{L\left(\epsilon^{-1}-1\right)+1} \geq 0,
$$


where the limit of zero noise in both quadratures is approached for $L \rightarrow 0$ and $\epsilon \rightarrow 1$. For example, with experimentally realisable parameters of $6 \mathrm{~dB}$ squeezing 19 and detection efficiency $\epsilon=0.95$ [21], one could obtain $S_{1}^{X}=0.25$ and $S_{1}^{Y}=0.05$, giving $S_{1}^{Y}+S_{1}^{X}=0.30$, compared to the limit of 2 implied by the uncertainty relation (9).

\section{Application to Quantum Spectroscopy}

Since the quadrature spectra calculated above apply to an in-loop field, which cannot be extracted using a beam splitter, it might seem that they have no physical significance. However, this is not the case. As I showed recently [12, placing a two-level atom in an squashed bath leads to linewidth narrowing of one atomic dipole quadrature, entirely analogous to that produced by a squeezed bath. The master equation is not identical, however, because the non-squashed quadrature is still shot-noise limited, so there is no line broadening of the other atomic dipole quadrature.

In this work I generalize the results of Ref. [12] to include light which is simultaneously squashed in both quadratures, or simultaneously squeezed and squashed. If the atom is coupled to the in-loop beam $b_{1}(t)$ with mode-matching $\eta$ then the atomic Hamiltonian is

$$
H(t)=-i\left[\sqrt{\eta} b_{1}(t)+\sqrt{1-\eta} \nu(t)\right] \sigma^{\dagger}(t)+\text { H.c. }
$$

Following the methods of Ref. [12], the expression for $b_{1}$ is modified from (26) by the addition of the atom's radiated field in the direction of the beam, $\sqrt{\eta} \tilde{\sigma_{x}}(\omega)$, to the input operator $\tilde{X}_{0}(\omega)$. Thus the total Hamiltonian can be written

$$
H(t)=H_{\mathrm{fb}}(t)+H_{0}(t),
$$

where $H_{0}(t)$ is as given in Eq. (12) and the Hamiltonian due to the feedback is

$$
\begin{aligned}
H_{\mathrm{fb}}(t)= & \lambda_{X} \frac{1}{2} \sigma_{y}(t)\left\{\sigma_{x}\left(t^{-}\right)+\left[X_{0}\left(t^{-}\right)+\sqrt{\theta_{X}} \xi_{X}\left(t^{-}\right)\right] \sqrt{\eta}\right\} \\
& +\lambda_{Y} \frac{1}{2} \sigma_{x}(t)\left\{\sigma_{y}\left(t^{-}\right)+\left[Y_{0}\left(t^{-}\right)+\sqrt{\theta_{Y}} \xi_{Y}\left(t^{-}\right)\right] \sqrt{\eta}\right\} .
\end{aligned}
$$

The feedback parameters are defined as

$$
\lambda_{X}=\frac{g_{X} \eta}{1-g_{X}} ; \quad \lambda_{Y}=\frac{g_{Y} \eta}{1-g_{Y}} .
$$

for the feedback of the homodyne current $I_{\text {hom }}^{Y}$ are defined analogously to those from the feedback of $I_{\mathrm{hom}}^{X}$.

In Eq. (35) the limit of broad-band feedback has been taken, with $\tilde{h}(\omega) e^{i \omega \tau}$ in Eq. (26) set to unity. This Markov approximation is justified provided the bandwidth of the feedback is very large compared to the characteristic rates of response of the system 22. In the present context the rate of atomic decay is unity so we require, for instance, $\tau \ll 1$. For a typical electro-optic feedback loop with a bandwidth in the $\mathrm{MHz}$ range, the atom would have to have be metastable to satisfy this inequality. The precise requirements for the validity of the Markov approximation will be investigated in a future publication. Of course even in the broad-band limit the feedback from the measurement of a particular part of the field must act after that part of the field has interacted with the atom. This is the reason for the use of the time argument $t^{-}$rather than $t$ in Eq. (35).

Now to describe the evolution generated by the total Hamiltonian (33), the theory of homodyne detection and feedback in the presence of white noise is required. This was first detailed in Ref. [23], generalizing the earlier work in Refs. [24] and [22]. The basic equation is

$$
d \rho(t)=\left\langle\exp \left[-i H_{\mathrm{fb}}(t) d t\right] \exp \left[-i H_{0}(t) d t\right] \rho(t) \exp \left[i H_{0}(t) d t\right] \exp \left[i H_{\mathrm{fb}}(t) d t\right]\right\rangle-\rho(t) .
$$

Here the ordering of the unitary operators has been chosen such that the time delay of the feedback has been taken into account and one can replace $t^{-}$in Eq. (35) by $t$.

In Eq. (37) the expectation value indicates an average over the bath operators $b_{0}(t)$ and $\nu(t)$, and the detector noise terms $\xi_{X}(t), \xi_{Y}(t)$. This is effected by making replacements such as

$$
\left[X_{0}(t) d t\right]^{2} \rightarrow L d t ; \nu(t) d t \nu^{\dagger}(t) d t \rightarrow d t .
$$


The result is

$$
\begin{aligned}
\dot{\rho}= & (1-\eta) \mathcal{D}[\sigma] \rho+\frac{\eta}{4 L} \mathcal{D}\left[(L+1) \sigma-(L-1) \sigma^{\dagger}\right] \rho \\
& -i \lambda_{X}\left[\frac{1}{2} \sigma_{y}, \frac{1}{2}\left\{(L+1) \sigma-(L-1) \sigma^{\dagger}\right\} \rho+\rho \frac{1}{2}\left\{(L+1) \sigma^{\dagger}-(L-1) \sigma\right\}\right] \\
& +i \lambda_{Y}\left[\frac{1}{2} \sigma_{x},-i \frac{1}{2}\left\{\left(L^{-1}+1\right) \sigma+\left(L^{-1}-1\right) \sigma^{\dagger}\right\} \rho+i \rho \frac{1}{2}\left\{\left(L^{-1}+1\right) \sigma^{\dagger}-\left(L^{-1}-1\right) \sigma\right\}\right] \\
& +\frac{\lambda_{X}^{2}\left(L+\theta_{X}\right)}{\eta} \mathcal{D}\left[\frac{\sigma_{y}}{2}\right] \rho+\frac{\lambda_{Y}^{2}\left(L^{-1}+\theta_{Y}\right)}{\eta} \mathcal{D}\left[\frac{\sigma_{x}}{2}\right] \rho .
\end{aligned}
$$

This again produces the atomic dynamics of Eqs. (15)-(17), but with

$$
\begin{aligned}
& \gamma_{x}=\frac{1}{2}\left[(1-\eta)+\eta L\left(1+\lambda_{X} / \eta\right)^{2}+\lambda_{X}^{2} \theta_{X} / \eta\right]=\frac{1}{2}\left[(1-\eta)+\eta S_{X}\right], \\
& \gamma_{y}=\frac{1}{2}\left[(1-\eta)+\eta L^{-1}\left(1+\lambda_{Y} / \eta\right)^{2}+\lambda_{Y}^{2} \theta_{Y} / \eta\right]=\frac{1}{2}\left[(1-\eta)+\eta S_{Y}\right], \\
& \gamma_{z}=\gamma_{x}+\gamma_{y}, C=1+\lambda_{X}+\lambda_{Y} .
\end{aligned}
$$

In the above equations, the introduction of the spectrum $S^{X}$ is based on Eq. (29), with the identification $\lambda_{X}=\eta g_{X} /\left(1-g_{X}\right)$, and similarly for $Y$. Note that the expressions for $\gamma_{x}$ and $\gamma_{y}$ depend upon the quadrature spectra (in the absence of the atom) in precisely the same way as those for pure squeezed (not squashed) light in Eqs. (18) and (19). This suggests that the natural explanation for the change in the decay rates is again the reduced fluctuations of the input light. It seems that squashed fluctuations are much the same as squeezed fluctuations as far as the atom is concerned. The one difference is that the constant $C$ in the equation of motion for $\left\langle\sigma_{z}\right\rangle$ is also altered by the feedback.

Consider now the case as in Sec. 3.4 where $L<1, \epsilon_{X}=0$ and $\epsilon_{Y}=\epsilon$. Then choosing $\lambda_{Y}=-\eta /(1+L \theta)$, so as to minimize the in-loop $Y$ quadrature spectrum, gives

$$
\begin{aligned}
\gamma_{x} & =\frac{1}{2}[(1-\eta)+\eta L], \\
\gamma_{y} & =\frac{1}{2}[(1-\eta)+\eta \theta /(1+L \theta)], \\
\gamma_{z} & =\gamma_{x}+\gamma_{y}, \quad C=1-\eta /(1+\theta L),
\end{aligned}
$$

where $\theta=\epsilon^{-1}-1$ as usual. Choosing $\epsilon=0.95$ and $L \approx 0.25$, as before, gives $\gamma_{z} \approx 1-0.85 \eta$. That is, in the limit of $\eta \rightarrow 1$, the rate of decay of the atomic population would be slowed by $85 \%$. In the ideal limit of $L \rightarrow 0$, and $\epsilon, \eta \rightarrow 1$, the atom would be frozen in its initial state and would not decay at all.

\section{Conclusion}

In this work I have presented for the first time the theory for a new class of in-loop light, namely light which may be both squashed (in either or both quadratures) and squeezed. Squeezing here refers to conventional quantum noise reduction, whereas squashing refers to the noise reduction produced by the feedback loop. Even without a squeezed input it is possible to reduce the noise in both quadratures of the in-loop field below the shot-noise limit. With a squeezed input it is possible, in principle, to reduce the noise in both quadratures to zero (by squeezing one and squashing the other).

I next derived the effect of this arbitrarily squeezed and squashed light on an in-loop atom. The calculated in-loop spectra precisely reflect the noise to which the atom responds, provided the bandwidth of the squeezing and the bandwidth of the feedback are much greater than the atomic linewidth. As the quantum fluctuations seen by the atom are reduced, the decay rates for the quadratures of the atom's dipole are reduced. In the limit that the atom is coupled only to in-loop light which is perfectly squeezed in one quadrature and perfectly squashed in the other, the atomic decay rates vanish and the atom's dynamics are frozen.

As discussed above, the main experimental difficulty with seeing squeezing-induced linenarrowing is related to efficiently coupling the squeezed light onto the atom. Using squashed light rather than squeezed light would not overcome this difficulty. However, highly squashed light should be easier to generate than highly squeezed light, because it is limited only by the homodyne detector efficiency. Also, it can be produced at any frequency for which a coherent source and 
the appropriate electro-optic equipment is available. These factors, plus the intriguing possibility of observing simultaneous linewidth narrowing on both atomic quadratures, suggest an important role for squashed light in experimental quantum spectroscopy.

\section{References}

[1] D.F. Walls, Nature 234, 210 (1986).

[2] J.G. Walker and E. Jakeman, Proc. Soc. Photo-Opt. Instrum. Eng. 492, 274 (1995).

[3] S. Machida and Y. Yamamoto, Opt. Commun. 57, 290 (1986).

[4] R.E. Slusher et al., Phys. Rev. Lett. 55, 2409 (1985).

[5] J.M. Shapiro et al, J. Opt. Soc. Am. B 4, 1604 (1987).

[6] Y. Yamamoto, N. Imoto and S. Machida, Phys. Rev. A 33, 3243 (1986).

[7] C.W. Gardiner, Quantum Noise (Springer, Berlin, 1991).

[8] D.F. Walls and G.J. Milburn, Quantum Optics (Springer, Berlin, 1994).

[9] H.-A. Bachor A Guide to Experiments in Quantum Optics (Wiley, Berlin, 1998).

[10] M.S. Taubman, H.M. Wiseman, D.E. McClelland, and H.-A. Bachor J. Opt. Soc. Am. B 12, $1792(1995)$.

[11] B.C. Buchler et al., Optics Letters 24, 259 (1999).

[12] H.M. Wiseman, Phys. Rev. Lett. 81, 3840 (1998).

[13] C.W. Gardiner, Phys. Rev. Lett. 56, 1917 (1986).

[14] C.M. Caves, Phys. Rev. D 23, 1693 (1981).

[15] H.P. Yuen, Phys. Rev. A 13, 2226 (1976).

[16] C.M. Caves and B.L. Schumaker, Phys. Rev. A 31, 3068 (1985).

[17] H.P. Yuen and J.H. Shapiro, IEEE Trans. IT 26, 78 (1980).

[18] Z. Ficek, P.D. Drummond, Phys. Today 50,34 (1997).

[19] Q.A. Turchette et al. Phys. Rev. A 58, 4056 (1998).

[20] J.J. Stefano, A.R. Subberud, and I.J. Williams, Theory and Problems of Feedback and Control Systems 2e (Mc Graw-Hill, New York, 1990).

[21] S. Schiller et al. Phys. Rev. Lett. 77, 2933 (1996).

[22] H.M. Wiseman, Phys. Rev. A 49, 2133 (1994); Errata ibid., 495159 (1994) and ibid. 50, 4428 (1994).

[23] H.M. Wiseman and G.J. Milburn, Phys. Rev. A 49, 4110 (1994).

[24] H.M. Wiseman and G.J. Milburn, Phys. Rev. Lett. 70, 548 (1993). 
Figure 1: Simplified diagram of the production of squashed light. The circle in the path of the in-loop beam is where an in-loop atom could be positioned. 